\title{
Images of Chest Diseases: a new format for Case Reports in Monaldi
}

When this Editorial team took over, one of our targets was the renewal of the Case Report Section. We intended to publish more Case reports with more interesting and clinical oriented iconography in order to attract the readers of Monaldi and increase the rate of "serial readers" to our Journal.

Over the years we have been consistent with our original commitment, as we published a number of Case Reports on various different aspects of Pulmonary Medicine, reported by authors of many different countries. However we feel that this task can be performed even better. Among the Case Reports submitted to Monaldi, there are many examples in which the picture/s (imaging, endoscopy, histology, etc.) would deserve publication but at the same time the accompanying text should be just a corollarium of the (beautiful and interesting, rare and unusual) images. This is the basic idea that is behind the beginning of this new format that we named "Images of Chest Diseases" and that we inaugurate in this issue of Monaldi. "Images" are not meant to substitute classic Case Reports, rather to be added to them to give our Authors another opportunity to submit their work to Monaldi.

Mondoni et al. report of a melanic "tattoo" on the trachea of a woman followed for a malignant melanoma. In this case, the CT, endoscopic and histologic images are self-explanatory, once more proving the concept that "seeing is believing"!

As a result we anticipate that other authors will send us their "Images of Chest Diseases", in the same format as this first example.

Editorial Board Monaldi Archives for Chest Diseases Pulmonary Series

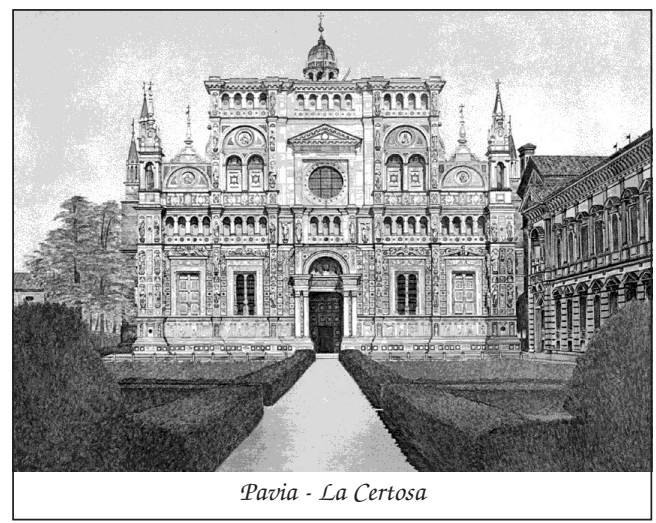

